Service social

\title{
Classes sociales et mouvements sociaux au Québec et au Canada, par David Descent, Louis Maheu, Martin Robitaille et Gilles Simard, Montréal, Saint-Martin, 1989, 206 pages.
}

\section{Gérald Doré}

Volume 39, numéro 2, 1990

Les problèmes sociaux

URI : https://id.erudit.org/iderudit/706487ar

DOI : https://doi.org/10.7202/706487ar

Aller au sommaire du numéro

Éditeur(s)

École de service social de l'Université Laval

ISSN

1708-1734 (numérique)

Découvrir la revue

Citer ce compte rendu

Doré, G. (1990). Compte rendu de [Classes sociales et mouvements sociaux au Québec et au Canada, par David Descent, Louis Maheu, Martin Robitaille et Gilles Simard, Montréal, Saint-Martin, 1989, 206 pages.] Service social, 39(2), 235-236. https://doi.org/10.7202/706487ar d'utilisation que vous pouvez consulter en ligne.

https://apropos.erudit.org/fr/usagers/politique-dutilisation/ 


\section{RECENSIONS}

\section{Classes sociales et mouvements sociaux au Québec et au Canada, par David DESCENT, Louis MAHEU, Martin ROBITAILLE et Gilles SIMARD, Montréal, Saint-Martin, 1989, 206 pages.}

Avant même d'ouvrir ce livre, on est frappé par l'à-propos de sa publication. II brise en effet la conspiration du silence (ou du refoulement culturel ?) qui, dans les années 1980, a entouré, au Québec, le thème des classes sociales, aussi bien dans le discours sociologique que dans le discours commun. Comme le souligne Louis Maheu, dans l'avant-propos, "ce n'est pas la première fois que ce corpus théorique est dit en crise [...] et vraisemblablement pas la dernière non plus [...] $C^{\prime}$ est ainsi que dans certaines conjonctures, ajoute-t-il, deviennent moments impératifs d'une démarche scientifique et la "négation " $d^{\prime}$ 'une forme théorique et sa reconstruction » (p. 11).

L'ouvrage se situe en quelque sorte en amont de ce processus de négation/reconstruction. II nous présente une bibliographie, précédée d'un essai de synthèse $d^{\prime}$ 'une partie de la documentation recensée. La bibliographie qui compte 120 pages est une compilation de livres et d'articles appartenant à la littérature canadienne et québécoise des sciences sociales, et principalement de la sociologie. Le texte le plus ancien remonte à 1950 (p. 118) et le plus récent est daté de 1987 (p. 183). C'est du moins ce dont on se rend compte en parcourant méthodiquement les titres de la bibliographie, qui livre plus que ce qu'annoncent les auteurs et l'éditeur, sur la jaquette et dans le texte même, en parlant de "quinze ans de la production en sciences sociales et en sociologie" (jaquette et p. 20) ou encore " des productions qui s'étendent de la fin des années 1960 jusqu'en 1986 » (p. 14 et 97). Comme il est de règle, on aurait pu s'attendre en fait à une définition claire de la période couverte par la bibliographie. Celle-ci est divisée en trois rubriques principales : classes sociales, État et rapports sociopolitiques, mouvements sociaux. La première rubrique comporte elle-même des subdivisions relatives aux différentes classes sociales. La rubrique de l'État traite de la question nationale, du développement régional, de l'accumulation et de la régulation sociale, de I'hégémonie et du bloc au pouvoir. Dans la dernière, les titres sont distribués par catégorie de mouvement social : féministe, pacifiste, écologique, etc.

L'essai qui précède la bibliographie est une synthèse de lecture à partir de plus de 300 des titres recensés. Bien qu'ils figurent en gras italique dans la bibliographie, ces titres sont repris dans une liste séparée, insérée entre l'essai et la bibliographie (p. 65-92); répétition dont l'utilité ne paraît pas évidente. Après quelques considérations générales, l'essai aborde le traitement du thème des classes sociales dans la documentation choisie, en procédant classe par classe : la bourgeoisie et ses diverses fractions, les classes moyennes, la classe ouvrière. Sous un titre dont le rapport au contenu n'est malheureusement pas assez explicite ("L'État, I'espace public et la société 
civile "), il passe ensuite en revue les pratiques conflictuelles qui se constituent en opposition à l'encadrement étatique et en rapport aux conditions de vie. A cet égard, l'essai nous offre des pages particulièrement éclairantes ( $p$. 44-48) pour la compréhension de la dynamique des mouvements sociaux répondant à ces caractéristiques. Les mouvements écologistes et pacifistes font ensuite l'objet d'un traitement spécifique, sous un titre encore inutilement abstrait ("Le rapport avec la nature et la domination culturelle et politique $»)$. La dernière section est consacrée au mouvement des femmes, sous un titre précis cette fois : "Le mouvement des femmes : d'un marché parallèle du travail au travail domestique et à une identité socioculturelle problématique ". La conclusion ramasse très bien l'essentiel du contenu de l'essai, en résumant successivement les apports de la sociologie canadienne et de la sociologie québécoise des dernières décennies à l'étude des classes sociales et des mouvements sociaux au Canada et au Québec.

On peut regretter qu'une section de l'essai n'ait pas été consacrée aux définitions, aux "grilles" des classes sociales, dans la documentation répertoriée. II se glisse bien quelques allusions à la question, au fil du texte (p. 19, 23-24, 31-32, 36, 41, 62), mais ce n'est pas suffisant pour entreprendre la " reconstruction » dont parle Maheu dans I'avant-propos. La même remarque vaut pour la définition des mouvements sociaux. L'articulation des deux, classes sociales et mouvements sociaux, aurait, elle aussi, mérité de faire I'objet d'une section séparée. Encore ici des allusions éparses (p. 12, 30-31, 34, $37,40-41,44-45)$, mais pas de traitement systématique qui puisse faire avancer la question. Ce flottement conceptuel explique sans doute l'étonnante localisation de la catégorie " jeunes" (p. 39-41), dans la section réservée à la classe ouvrière. Ou encore la simplification qui consiste à donner une référence sur les groupes populaires comme illustration des pratiques conflictuelles reliées à "l'encadrement étatique de la marginalité et de la délinquance " (p. 44). Ou encore l'indécision entre "classes moyennes " et " petites bourgeoisies » pour la dénomination des classes intermédiaires (p. 31 et 115).

Malgré ses limites, ce livre n'en reste pas moins un ouvrage de référence de première main. En feront leur profit les chercheurs et chercheuses, professeurs et professeures, étudiants et étudiantes en sciences sociales pour qui les classes sociales et les conflits sociaux méritent d'être étudiés, parce qu'ils continuent d'exister; malgré le discours d'une certaine sociologie, par trop tributaire de l'idéologie rétrolibérale, de son individualisme et de sa visée consensuelle intéressée (concertation, partenariat, etc.). Signe des temps ? Fernand Dumont, qui n'a guère abordé ce sujet depuis les années 1960 (p. 21, 73, 101), affirmait vouloir réactualiser la "difficile question des classes sociales", lors du colloque organisé par l'Institut québécois de recherche sur la culture, à l'occasion de son dixième anniversaire, en 1989.

Gérald Doré

École de service social Université Laval 